\title{
Acceleration-critical density time-delay model for crowd stability analysis based on Lyapunov theory
}

\author{
Rongyong Zhao, Ping Jia ${ }^{*}$, Yan Wang, Cuiling Li, Yunlong Ma, and Zhishu Zhang \\ School of Electronic and Information Engineering, Tongji University, Shanghai, China
}

\begin{abstract}
Crowd stability analysis is one of research hotspots to alleviate the severe situation of stampede accidents worldwide. Different from the conventional analysis models for crowd stability based on pedestrian density, this study analyses the characteristics of external disturbances and internal obstacle disturbance based on Lyapunov's theory. The critical range of crowd acceleration in crowd evacuation is obtained, a crowd merging acceleration-critical density time delay model is established, and a stability criterion of acceleration vector based on Lyapunov is obtained based on Lyapunov stability analysis. This provides new information for ensuring the stability of crowd movement in public places, assessing the stability of the crowd in the area, and taking reasonable protection and guidance measures prior to instability of a crowd flow.
\end{abstract}

Keywords: Crowd stability analysis, External disturbances, Internal obstacle disturbance.

\section{Introduction}

Serious pedestrian accidents occurred frequently in the past decade. For example, on June 20, 2013, Beckham visited Tongji University. When he entered the stadium, the fans lost control and the stampede caused huge casualties. On December 31st, 2014, a large number of pedestrians under the observation deck of the Bund in Shanghai hedged up and down, causing 36 pedestrians to die and 49 others to be injured. These accidents around the world caused a large number of casualties and economic losses.

In general, the crowd density in a specific area in public places can reflect the stability of the crowd to a large extent, that is, the crowd density can be used as an indicator of crowd stability [1]. Therefore, it is particularly important to obtain the crowd density in a specific area of public places, evaluate the stability of the crowd in this area, and take reasonable guidance measures in time before the crowd is in a state of instability. Different from the conventional analysis models above, this study analyzes the characteristics of external disturbances and internal obstacle disturbance based on Lyapunov's theory, then proposes a crowd merging acceleration-critical density time delay model.

\footnotetext{
*Corresponding author:2033108@tongji.edu.cn
} 


\section{The stability of crowd evacuation based on Lyapunov}

Stability refers to the performance of the system that is restored from the initial deviation state to the original equilibrium state after being disturbed. Any system may deviate from the original equilibrium state under a disturbance with sufficient energy, resulting in an initial deviation. The traditional stability criterion methods of systems are classified as: Helwitz stability criterion, Routh stability criterion, root locus analysis method, Nyquist stability criterion and Lyapunov stability criterion. The moving crowd can also be regarded as a system. The stability of the crowd system reflects the ability of the crowd to return to a normal and stable state of movement when encountering an emergency or sudden situation [2] (The external disaster disturbance of the crowd system (such as fire smoke, poisonous gas, heat waves, earthquakes, violent terrorist attacks, etc.) and internal disturbances (such as pedestrians falling, crowding of pedestrians or large luggage falling, etc.) will affect the stability of the crowd merging system.

Lyapunov stability uses a state vector to describe the stability of the system, which can effectively solve the stability problem of nonlinear systems [3]. The stability of Lyapunov is mainly aimed at studying the equilibrium state of the system. For all time $t$, as long as it is satisfied:

$$
\dot{\mathrm{x}}_{\mathrm{eq}}=\mathrm{f}_{\mathrm{l}}\left(\mathrm{x}_{\mathrm{eq}}, \mathrm{t}\right)=0
$$

The state of $x_{e q}$ is called the equilibrium state. When the system is in equilibrium, its components no longer change with respect to time.

Assuming that the initial state of the system is in the closed-sphere domain $\mathrm{S}(\delta)$, the closed-sphere domain is based on the equilibrium state $x_{e q}$ as the center of the sphere and $\delta$ as the radius, that is

$$
|| \mathrm{x}_{0}-\mathrm{x}_{\mathrm{eq}}|| \leq \delta, \mathrm{t}=\mathrm{t}_{0}
$$

If in the process of $t \rightarrow \infty$, the solution $x\left(t ; x_{0}, t_{0}\right)$ of the system equation is located in the closed sphere domain $\mathrm{S}(\varepsilon)$, the closed sphere domain $\mathrm{S}(\varepsilon)$ is the equilibrium state $x_{e q}$ as the center of the sphere and $\delta$ as the radius which is

$$
\left\|x\left(t ; x_{0}, t_{0}\right)-x_{0}\right\| \leq \varepsilon, \quad t \geq t_{0}
$$

It is said that the equilibrium state $x_{e q}$ of the system is stable in the sense of Lyapunov[4].

\section{The acceleration-critical density time-delay model}

According to the Lyapunov stability theory, the stability criterion of the acceleration vector of the crowd flow is analyzed. The macroscopic model of the flow of panic crowds at merging intersections is composed of Eqs. (4)-(6).

$$
\begin{gathered}
\frac{\partial \rho}{\partial \mathrm{t}}+\frac{\partial(\rho \mathrm{v})}{\partial \mathrm{x}}+\frac{\partial(\rho \mathrm{u})}{\partial \mathrm{y}}=0 \\
\frac{\partial \mathrm{v}}{\partial \mathrm{t}}+\mathrm{v} \frac{\partial \mathrm{v}}{\partial \mathrm{x}}=-\frac{1}{\rho} \frac{\partial \mathrm{P}_{\mathrm{h}}}{\partial \mathrm{t}}+\frac{1}{\tau}\left(\mathrm{V}_{\mathrm{e}}-\mathrm{v}\right) \\
\frac{\partial \mathrm{u}}{\partial \mathrm{t}}+\mathrm{u} \frac{\partial \mathrm{u}}{\partial \mathrm{y}}=-\frac{1}{\rho} \frac{\partial \mathrm{P}_{\mathrm{v}}}{\partial \mathrm{t}}+\frac{1}{\tau}\left(\mathrm{U}_{\mathrm{e}}-\mathrm{u}\right)
\end{gathered}
$$


where $\rho$ is the density of the evacuated crowd, and $v$ and $u$ are the horizontal and vertical velocities at time $t$ at $(x, y) . V_{e}$ and $U_{e}$ are the equilibrium speeds in the horizontal and vertical directions, respectively, and are given by the steady-state relationship between the speed and density of the flowing group. $P_{h}$ and $P_{v}$ are the pressure terms in the horizontal and vertical directions, respectively, and $\tau$ is the relaxation time coefficient, which is the time constant for adjusting the speed of the flowing group to the equilibrium speed.

The equilibrium flow of the macro-crowd flowing model of panic spread at merging junctions is $q_{e q}(x, y, t)=\left[\rho_{e q}(x, y, t), v_{e q}(x, y, t), u_{e q}(x, y, t)\right]^{T}$, The panic flow is $q_{p}(x, y, t)$, Among them, $\rho(x, y, t)$ is the density of evacuated people, $v(x, y, t)$, $u(x, y, t)$ are the horizontal and vertical directions at time $t$ at $(x, y)$ speed. If the spatial gradient of the panic state is bounded, the spread of the crowd flow $\mathrm{q}(x, y, t)$ in the panic state is stable, that is,

$$
\left\|\frac{\partial_{\mathrm{qp}}}{\partial_{\mathrm{x}}}(\mathrm{x}, \mathrm{y}, \mathrm{t})\right\|<\infty \text { and }\left\|\frac{\partial_{\mathrm{qp}}}{\partial_{\mathrm{y}}}(\mathrm{x}, \mathrm{y}, \mathrm{t})\right\|<\infty .
$$

At any time $t>0,(x, y) \in M, M$ is the set of the entire junction area. In addition to the above, when $\lim _{t \rightarrow \infty}\left\|\frac{\partial_{q_{p}}}{\partial_{x}}(x, y, t)\right\|=0$ and $\lim _{t \rightarrow \infty}\left\|\frac{\partial_{q_{p}}}{\partial_{y}}(x, y, t)\right\|=0$, the propagation of equilibrium flow $q_{e q}(x, y, t)$ is asymptotically stable [5].

Expand the partial derivatives in formulas (4), (5) and (6), $\rho(x, y, t), v(x, t)$ and $\mathrm{u}(\mathrm{y}, \mathrm{t})$ are expressed by total differential, and then the variable $\rho_{t}, \rho_{x, y}, v_{t}, v_{x}, u_{t}, u_{y}$, are extracted, and using the matrix, the values $v_{c}$ and $u_{c}$ of the characteristic velocity in the horizontal and vertical directions are:

$$
\begin{gathered}
\mathrm{v}_{\mathrm{c}}=\frac{\mathrm{dx}}{\mathrm{dt}}=\mathrm{v}+\frac{1}{2 \rho} \frac{\partial \mathrm{P}_{\mathrm{h}}}{\partial \mathrm{v}} \pm \sqrt{\frac{1}{4 \rho^{2}}\left(\frac{\partial \mathrm{P}_{\mathrm{h}}}{\partial \mathrm{v}}\right)^{2}+\frac{\partial \mathrm{P}_{\mathrm{h}}}{\partial \rho}} \\
\mathrm{u}_{\mathrm{c}}=\frac{\mathrm{dy}}{\mathrm{dt}}=\mathrm{u}+\frac{1}{2 \rho} \frac{\partial \mathrm{P}_{\mathrm{v}}}{\partial \mathrm{u}} \pm \sqrt{\frac{1}{4 \rho^{2}}\left(\frac{\partial \mathrm{P}_{\mathrm{v}}}{\partial \mathrm{y}}\right)^{2}+\frac{\partial \mathrm{P}_{\mathrm{v}}}{\partial \rho}}
\end{gathered}
$$

Consider a considerable disturbance near the equilibrium density and average velocity. Suppose that the model has a constant evacuation density $\rho_{0}=\rho_{e}$ and velocity $v_{0}=$ $V_{e}\left(\rho_{e}\right), u_{0}=U_{e}\left(\rho_{e}\right)$. The disturbances during the flow are:

$$
\xi=(\vec{x}, \vec{y})-\vec{X}(t)-\vec{Y}(t)
$$

where $(\vec{x}, \vec{y})(\mathrm{t})$ is the position of the evacuated group at time $t$.

According to the characteristics of internal disturbance and external disturbance, the external disturbance can be set as:

$$
\begin{gathered}
\xi_{\text {fire }}=(\vec{x}, \vec{y})_{\text {fire }}-\vec{X}_{\text {fire }}(t)-\vec{Y}_{\text {fire }}(t) \\
\xi_{p g}=(\vec{x}, \vec{y})_{p g}-\vec{X}_{p g}(t)-\vec{Y}_{p g}(t) \\
\xi_{\text {exte }}=\xi_{\text {fire }}+\xi_{\text {equ }}+\cdots+\xi_{\text {pg }}
\end{gathered}
$$

where $\xi_{\text {fire }}$ represents the interference caused by the fire smoke, $\xi_{\text {equ }}$ represents the interference caused by the earthquake, $\xi_{p g}$ represents the interference caused by the poisonous gas, $\xi_{\text {exte }}$ stands for external disturbance. For example, the energy impact of earthquakes on the crowd is $30 \% \sim 50 \%$, the energy impact of poisonous gas on the crowd is 
$10 \% \sim 20 \%$, the energy impact of violent terrorist attacks on the crowd is $30 \% \sim 40 \%$, the energy impact of fire \& smoke on the crowd is $10 \% \sim 30 \%$.

An internal disturbance can be set as:

$$
\xi_{\text {inte }}=\xi_{\text {ped }}+\cdots+\xi_{\text {cop }}
$$

where $\xi_{\text {ped }}$ represents the interference caused by the pedestrians falling, $\xi_{\text {cop }}$ represents the interference caused by the crowding of pedestrians, $\xi_{\text {inte }}$ stands for internal disturbance.

$$
\xi_{\text {total }}=\xi_{\text {exte }}+\xi_{\text {inte }}
$$

where $\xi_{\text {total }}$ stands is total disturbance.

According to Eqs. (7) and (8), derivations of $X(t)$ and $Y(t)$ as $\dot{X}(t), \dot{Y}(t)$.

$$
\begin{gathered}
\dot{X}(\mathrm{t})=\mathrm{v}_{0}+\left.\frac{1}{2 \rho_{0}} \frac{\partial \mathrm{P}_{\mathrm{h}}}{\partial \mathrm{v}}\right|_{\left(\rho_{0}, \mathrm{v}_{0}\right)} \pm \sqrt{\frac{1}{4 \rho_{0}^{2}}\left(\left.\frac{\partial \mathrm{P}_{\mathrm{h}}}{\partial \mathrm{v}}\right|_{\left(\rho_{0}, \mathrm{v}_{0}\right)}\right)^{2}+\left.\frac{\partial \mathrm{P}_{\mathrm{h}}}{\partial \rho}\right|_{\left(\rho_{0}, \mathrm{v}_{0}\right)}} \\
\dot{\mathrm{Y}}(\mathrm{t})=\mathrm{u}_{0}+\left.\frac{1}{2 \rho_{0}} \frac{\partial \mathrm{P}_{\mathrm{v}}}{\partial \mathrm{u}}\right|_{\left(\rho_{0}, \mathrm{u}_{0}\right)} \pm \sqrt{\frac{1}{4 \rho_{0}^{2}}\left(\left.\frac{\partial \mathrm{P}_{\mathrm{v}}}{\partial \mathrm{u}}\right|_{\left(\rho_{0}, \mathrm{u}_{0}\right)}\right)^{2}+\left.\frac{\partial \mathrm{P}_{\mathrm{v}}}{\partial \rho}\right|_{\left(\rho_{0}, \mathrm{u}_{0}\right)}}
\end{gathered}
$$

Considering $\xi$ and expand with a power series to expand the horizontal equalization speed $V_{e}(\rho, v)$ and the vertical equalization speed $U_{e}(\rho, u)$.

$$
\begin{aligned}
& \mathrm{V}_{\mathrm{e}}(\rho, \mathrm{v})=\mathrm{V}_{\mathrm{e}}^{0}+\xi\left[\left(\mathrm{V}_{\mathrm{e}}\right)_{\rho}^{0} \rho_{1}(\mathrm{t})+\left(\mathrm{V}_{\mathrm{e}}\right)_{\mathrm{v}}^{0} \mathrm{v}_{1}(\mathrm{t})\right]+\cdots \\
& \mathrm{U}_{\mathrm{e}}(\rho, \mathrm{u})=\mathrm{U}_{\mathrm{e}}^{0}+\xi\left[\left(\mathrm{U}_{\mathrm{e}}\right)_{\rho}^{0} \rho_{1}(\mathrm{t})+\left(\mathrm{U}_{\mathrm{e}}\right)_{\mathrm{u}}^{0} \mathrm{u}_{1}(\mathrm{t})\right]+\cdots
\end{aligned}
$$

According to the equations above, the horizontal direction can be obtained

$$
\begin{gathered}
-\rho_{1} \mathrm{v}_{\mathrm{x}}+\rho_{0} \mathrm{v}_{1}=0 \\
\rho_{0} \dot{\rho_{1}}+\mathrm{v}_{2}\left(\left.\frac{\partial \mathrm{P}_{\mathrm{h}}}{\partial \mathrm{v}}\right|_{\left(\rho_{0}, \mathrm{v}_{0}\right)}-\rho_{0} \mathrm{v}_{\mathrm{x}}\right)+\left.\rho_{2} \frac{\partial \mathrm{P}_{\mathrm{h}}}{\partial \rho}\right|_{\left(\rho_{0}, \mathrm{v}_{0}\right)}+\rho_{1} \mathrm{v}_{1}\left(\left.2 \frac{\partial \mathrm{P}_{\mathrm{h}}}{\partial \rho \partial \mathrm{v}}\right|_{\left(\rho_{0}, \mathrm{v}_{0}\right)}-\mathrm{v}_{\mathrm{x}}\right)+ \\
\mathrm{v}_{1}^{2}\left(\rho_{0}+\left.\frac{\partial \mathrm{P}_{\mathrm{h}}}{\partial \mathrm{v} \partial \mathrm{v}}\right|_{\left(\rho_{0}, \mathrm{v}_{0}\right)}\right)+\left.\rho_{1}^{2} \frac{\partial \mathrm{P}_{\mathrm{h}}}{\partial \rho \partial \rho}\right|_{\left(\rho_{0}, \mathrm{v}_{0}\right)}+\frac{1}{\tau}\left[\mathrm{v}_{1}-\left(\mathrm{V}_{\mathrm{e}}\right)_{\rho}^{0} \rho_{1}-\left(\mathrm{V}_{\mathrm{e}}\right)_{\mathrm{v}}^{0} \mathrm{v}_{1}\right]=0
\end{gathered}
$$

According to Eqs. (14) and (15), the linear relationship between density and speed can be obtained.

To get:

Where $v_{1}(0)$ is the initial velocity value at time 0 .

This model has two different characteristic velocities in the horizontal direction, one velocity is $v_{0}+v_{x 1}\left(>v_{0}\right)$, the other velocity $v_{0}+v_{x 2}\left(<v_{0}\right), v_{x 1}$ and $v_{x 2}$ are two of $v_{x}$ calculated according to formula (10) value.

For the second-order macroscopic crowd evacuation model, the velocity $v_{0}+$ $v_{x 1}\left(>v_{0}\right),\left.\quad \frac{\partial P_{h}}{\partial v}\right|_{\left(\rho_{0}, v_{0}\right)}=0,\left.\frac{\partial V_{e}}{\partial v}\right|_{\left(\rho_{0}, v_{0}\right)}=0$ and $\left.\frac{\partial V_{e}}{\partial \rho}\right|_{\left(\rho_{0}, v_{0}\right)}<0$, so

$$
\alpha_{\mathrm{v}}=\frac{1}{2 \tau}\left(1+\frac{\left|\left(\mathrm{v}_{\mathrm{e}}\right)_{\rho}^{0}\right| \rho_{0}}{\mathrm{v}_{\mathrm{x}}}\right) \gg 1, \beta_{\mathrm{v}}>0
$$

The amplitude of this velocity disturbance quickly decays to zero. Therefore, for this model, the influence of the perturbed forward motion branch can be ignored.

Consider another effect of speed on stability:

$$
P_{h}=\frac{|v+u|^{\tau_{v i j}} \rho^{\gamma+1}}{\beta-\rho^{\gamma+1}}
$$




$$
\mathrm{v}_{\mathrm{x}}=-\sqrt{-\frac{\mathrm{v}_{\mathrm{e}}^{\prime}\left(\rho_{0}\right)}{2 \tau}}
$$

Substituting $P_{h}$ and $v_{x}$ with Eqs (23) and (24) to obtain $\alpha_{v}$, and further obtain the stable critical value of the velocity in the horizontal direction.

$$
\begin{gathered}
\alpha_{\mathrm{v}}=\frac{1}{2 \tau}\left(1-\rho_{0} \sqrt{-2 \mathrm{~V}_{\mathrm{e}}^{\prime}\left(\rho_{0}\right) \tau}\right) \\
\frac{1}{2} \geq-\rho_{0}^{2} \mathrm{~V}_{\mathrm{e}}^{\prime}\left(\rho_{0}\right) \tau
\end{gathered}
$$

In the same way, the vertical direction can be obtained according to the above formula:

$$
\mathrm{u}_{1}(\mathrm{t})=\frac{\alpha_{\mathrm{u}}}{\beta_{\mathrm{u}}} \frac{\mathrm{e}^{-\alpha \mathrm{t}}}{\left(1+\frac{\alpha}{\beta \mathrm{u}_{1}(0)}\right)-\mathrm{e}^{-\alpha \mathrm{t}}}
$$

where $u_{1}(0)$ is the initial speed value at $t=0$.

We can use the results obtained above to calculate the critical value of the vertical velocity of the model.

$$
\alpha_{\mathrm{u}}=\frac{1}{2 \tau}\left(1+\frac{\left|\left(\mathrm{U}_{\mathrm{e}}\right)_{\rho}^{0}\right| \rho_{0}}{\mathrm{u}_{\mathrm{y}}}\right) \gg 1, \beta_{\mathrm{u}}>0
$$

Consider the influence of another speed on stability, and further obtain the stability critical value of the vertical direction of the speed. The critical value of the magnitude and direction of the velocity differential is [6]:

$$
\begin{gathered}
\left\|v^{\prime}\right\|=\sqrt{V_{e}^{\prime 2}+U_{e}^{\prime 2}} \leq \frac{1}{2 \rho_{0}^{2} \tau} \\
\theta=\arctan \frac{U_{e}^{\prime}}{V_{e}^{\prime}}
\end{gathered}
$$

where $\rho_{0}$ is the initial velocity of the crowd, $\theta$ represents the direction of the crowd acceleration, and $\tau$ is the velocity characteristic parameter.

After stability analysis, the critical range of crowd acceleration in crowd evacuation is obtained, the crowd merging acceleration-critical density time lag model is established, and the stability criterion of acceleration vector based on Lyapunov's crowd regional flow is obtained[7]. When the crowd acceleration value exceeds the critical value $\frac{1}{2 \rho_{0}^{2} \tau}$, the crowd movement is unstable and stampede events are prone to occur; when the crowd acceleration value is less than the critical value, the crowd movement is in a stable state.

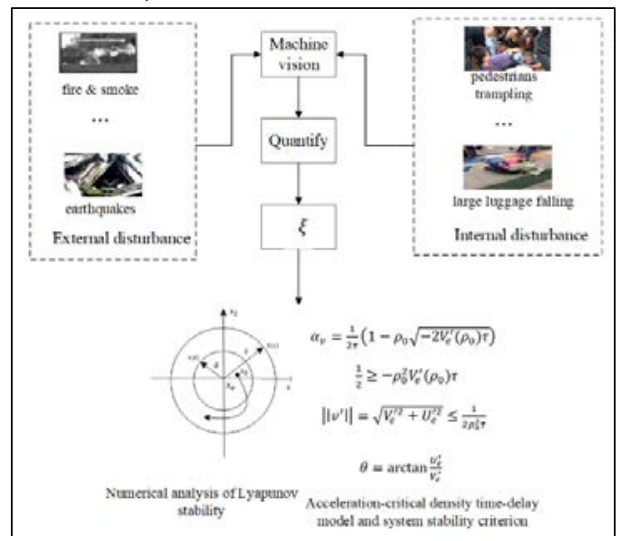

Fig. 1. Acceleration-critical density time-delay model for system stability criterion. 
As shown in Figure 1, when an emergency occurs in the crowd, the disturbance factor $\xi$ is quantified by machine vision. The acceleration value of the crowd is calculated based on Lyapunov's criterion of the stability of the acceleration vector of the flow of the crowd. After calculating acceleration value of the crowd, the stable state of the crowd is analysed.

\section{Summary}

In this study, Lyapunov is used to establish a crowd merging acceleration-critical density time lag model, and the stability criterion of the acceleration vector based on Lyapunov's crowd area flow is obtained. When the crowd acceleration is less than the critical value $\frac{1}{2 \rho_{0}^{2} \tau}$, the crowd movement is in a stable state. In addition, when the acceleration value of the crowd exceeds the critical value, the movement of the crowd is unstable, and a stampede event is very prone to occur. Therefore, the establishment of a crowd merging accelerationcritical density time lag model is of great significance for assessing the stability of the crowd in the area and taking preventive measures.

This research is financially supported by the National Natural Science Foundation of China (No.72074170).

\section{References}

1. Hongquan S, Xuejun L, Guonian L, et al. Video Scene Invariant Crowd Density Estimation Using Geographic Information Systems [J]. Wireless Communication over ZigBee for Automotive Inclination Measurement. China Communications, 2014, 11(11):80-89.

2. Yongxiang Zhao, Tuantuan Lu, Libi Fu, Peng Wu, Meifang Li. Experimental verification of escape efficiency enhancement by the presence of obstacles[J]. Safety Science, 2020,122.

3. Computation - Neural Computation; Studies from Huazhong University of Science and Technology in the Area of Neural Computation Reported (Principled Reward Shaping for Reinforcement Learning Via Lyapunov Stability Theory)[J]. Journal of Robotics \& Machine Learning,2020.

4. Kecai Cao, Yangquan Chen, Dan Stuart, Dong Yue. Cyber-physical Modeling and Control of Crowd of Pedestrians: A Review and New Framework[J].IEEE/CAA Journal of Automatica Sinica,2015,2(03):334-344.

5. Jingang Yi, Hao Lin, Luis Alvarez, Roberto Horowitz. Stability of macroscopic traffic flow modeling through wavefront expansion[J]. Transportation Research Part B,2003,37(7).

6. Qianshan Hu. Stability Analysis of Panic Crowd Evacuation at T-shaped Street Junction[D]. Shanghai: Tongji University. 2019.

7. J Zhang, W Klingsch, A Schadschneider, A Seyfried. Transitions in pedestrian fundamental diagrams of straight corridors and T-junctions [J]. Journal of Statistical Mechanics: Theory and Experiment, 2011, 2011(6). 